\title{
A Novel Captive Portal System for Reduction of Wireless Networks Security Threats in the Tamale Metropolis
}

\author{
Sakara S. \\ University for Development \\ Studies Faculty of \\ Mathematical Sciences \\ Department of Computer \\ Science, \\ Navrongo, Ghana
}

\author{
Alhassan A. \\ University for Development \\ Studies Faculty of \\ Mathematical Sciences \\ Department of Computer \\ Science, \\ Navrongo, Ghana
}

\author{
Alhassan S. \\ University for Development \\ Studies Faculty of \\ Mathematical Sciences \\ Department of Computer \\ Science, \\ Navrongo. Ghana
}

\begin{abstract}
This research work provides an entire overview of Wireless Local Area Network (WLAN), its structure or components and the security threats associated with it. Review of literature and survey of institutions and businesses in the Tamale Metropolis, shows that Wireless Local Area Network is commonly used especially by hospitals, schools, internet cafes and some other private organizations. Its main's advantage to the society is that a wireless network allows machines to be fully mobile as long as they remain in radio range and also avoids the burden of having cables between the machines. However, Wireless Local Area Networks with these advantages comes with the security threats. The research therefore evaluates the advantages and disadvantages of Wireless Local Area Networks, determine the structure/components of Wireless Local Area Networks and the security threats associated with it. It also revealed that Wireless Local Area Networks must be protected or the security of wireless networks must be enhanced. The research therefore developed a web-based application (a captive portal) to help reduce the security threats associated with Wireless Local Area Network, by preventing intruders or hackers into the system through; capturing user credentials, masquerading as an authorized Access Point by beaconing the WLAN's service set identifier (SSID) and capturing data to recover a Wired Equivalent Privacy (WEP) key.
\end{abstract}

\section{General Terms}

Administrator, User, Wireless Local Area Network, Security threats, Captive Portal.

\section{Keywords}

Bootstrap (JavaScript, CSS), Structured query Language (SQL), Hypertext markup language (HTML), Adobe Dreamweaver, Personal Home Page (PhP), web-based, Proposed system, Wireless Local Area Network (WLAN).

\section{INTRODUCTION}

Wireless Local Area Network is a type of network which a user can have a high bitrate network connection through a wireless (radio) connection. The WLAN networks are usually high in bitrate but relatively short in range. There are several standards that specify the different kinds of WLANs. The Institute of Electrical and Electronics Engineers (IEEE) standards for Wireless Local Area Network (WLAN) also include an encryption algorithm called Wired Equivalent Privacy (WEP). Its purpose was originally to make Wireless Local Area Network (WLAN) connections as safe as regular Local Area Network connections (Kujala, 2003). In Ghana, NetSol brings to the table over years to combined network infrastructure experience. Their portfolio can provide the robust, secure connectivity, whether dealing with a single office or several offices in a company. NetSol believes that the key to delivering a solid wireless network set up or configuration (NetSol, 2007). Wireless networking enables the same capabilities and comparable speeds of a wired 10BASE-T network without the difficulties associated with laying wire, or stringing Ethernet cables throughout an office building or home. Every room in a wireless home or office can be "connected" to the network, so adding more users and growing a network can be as simple as installing a new wireless network adapter (Whitepapers, 2008). A captive portal system redirects any user or customer who connects to your wireless to an authentication page before they are allowed to access the Wireless Network (internet). The captive portal technique forces an HTTP client on a network to see an authentication page before using the Internet normally. This is done by intercepting all packets, regardless of address or port, until the user opens a browser and tries to access the Internet. Below are some benefits of captive portals:

- Allows you to manage and bill your Wi-Fi users

- It helps protect against hackers

- Portals Allow for Customizable Usage Plans

\section{MATERIALS AND METHODS}

The study makes use of only primary and secondary data. This data was sourced by interviews, Semi-Structured Interview, observations and Library Research in the Tamale metropolis. The proposed system consists of the Interactive layer, Logic layer, Access layer and Storage layer. Technology/tools used in developing the system were Personal Home Page (PHP), Structured Query Language (SQL), Hypertext markup language (HTML), Adobe Dreamweaver and Bootstrap. The designed architecture of the Web-based application is to reduce the security threats associated with Wireless Local Area Network.

\subsection{Interactive Layer}

The interactive phase is the interface between the user and the system/administrator, which directs the user in terms of where to go and what to do depending on the specific kind of user. This phase provides separate tasks for user and the administrator.

\subsubsection{Users}

Based on the privileges level, the system allows three different kinds of users to sign up or login to the system.

\subsubsection{Administrator}

This is where the entire system is managed, which includes the management and information of all the users on the 
proposed system, and the maintenance of the proposed system and its resources.

\subsection{Logic Layer}

This manages the various services of the system, it receives request from the interactive layer, process it and pass it down to the access layer and further down to the storage layer. The result is sent back to the presentation layer. The two modules in the logic layer that interact within the system are signup new user and login.

\subsection{Access Layer}

The Access Layer is located in the database server and contains the data processing logic in the database server. Its main task is to receive requesting information from the web server, then complete the function of querying, refreshing and changing data in the database. Thereafter, it returns the results to the web server.

\subsection{Storage Layer}

Storage (database) constitutes a core element of the proposed system. In addition processed data concerning new member signup and instructors are managed in the database. The database management system (DBMS) used is MySQL embedded inside Xampp server. The database design incorporates the tables of records and the relationships among tables. Tables in the proposed system are just information tables.

\subsection{Personal Home Page (PHP)}

PHP is the most well-known scripting language for enhancing web pages. It was used in developing this system because it's easier to learn and quicker to create a website with PHP compared to ASP.NET. It is open source and works on almost all operating systems. Unlike ASP.NET, Error handling can be performed greatly and easily and error descriptions and messages are cleared explained. PHP can be run on free web servers such as Linux and Apache.

\subsection{Structured Query Language (SQL)}

SQL is a very well-known and powerful open source database and data storage system. It was used best it's a best choice for PHP, the selected programming language to develop unique website because it facilitates to connect to and manage MySQL database. Information and data can be retrieved quickly and easily and used by multiple website users at the same time.

\subsection{Bootstrap (CSS, HTML, JavaScript)}

Bootstrap is the most popular HTML, CSS, and JavaScript framework for developing responsive, mobile first projects on the web. Bootstrap allows for mobile view, this makes the system viewable on a mobile device. We don't code bootstrap, it is a plugin that we download and call it in any page that its need when coding in PHP language. HTML in bootstrap was used to design and develop the interface of the system (i.e. both the login interface and the signup interface) and CSS in bootstrap was used to style the web page (i.e. the background color, font size, font style and etc.). With Bootstrap, you get extensive and beautiful documentation for common HTML elements, dozens of custom HTML and CSS components, and awesome jQuery plugins.

\subsection{Adobe Dreamweaver}

Adobe Dreamweaver is an application used by web designers and developers to create websites and applications for use across multiple targets. Adobe Dreamweaver as a third party software was used in the developing the system because it supports PHP and MySQL.

\section{IMPLEMENTATION AND RESULTS}

The proposed system is an external Captive Portal system which has in it all the necessary functionalities of a captive portal and more. This system is a PHP web application which stores user credentials in a MySQL database. It is different from the existed captive portals in the sense that it has a sign up page, where clients need to sign up with their credentials which will be stored in a MySQL Database. When clients or users sign up to the system, a link is sent to the administrator's email to verify and grant the user access to the wireless network. The proposed system was implemented on a Core i5-4210U Intel microprocessor with Xampp Server v3.2.2 and Adobe Dreamweaver CS6. The program codes were written in PHP and HTML. The figures below are web pages which indicate how the developed system works.

\subsection{Homepage of the Proposed System}

This is the interface displayed when the system is installed and run. This is the SIGNUP page, the user needs to sign up to the system by filling in the required credentials; username, email and password.

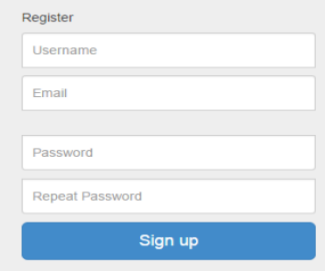

Figure 1: System Signup

\subsection{System Login of the Proposed System}

The Login interface that give access to the system depending on the access level. When a user registers and verified by the administrator, the user is then given access to the system by logging in with the his username and password

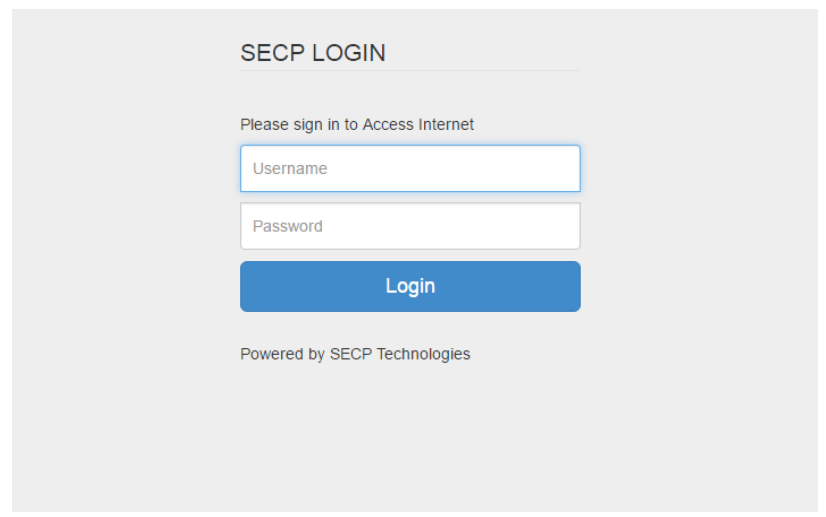

Figure 2: System Login

\subsection{System Error Login Page}

The Error Login Page displays an error message (i.e. Wrong Username or Password) when a user inputs an invalid username and password. When a client/user enters an either 
wrong username or password, the page displays the same error message "Wrong Username or Password".

\subsection{System Error Login Page}

The Error Login Page displays an error message (i.e. Wrong Username or Password) when a user inputs an invalid username and password. When a client/user enters an either wrong username or password, the page displays the same error message "Wrong Username or Password".

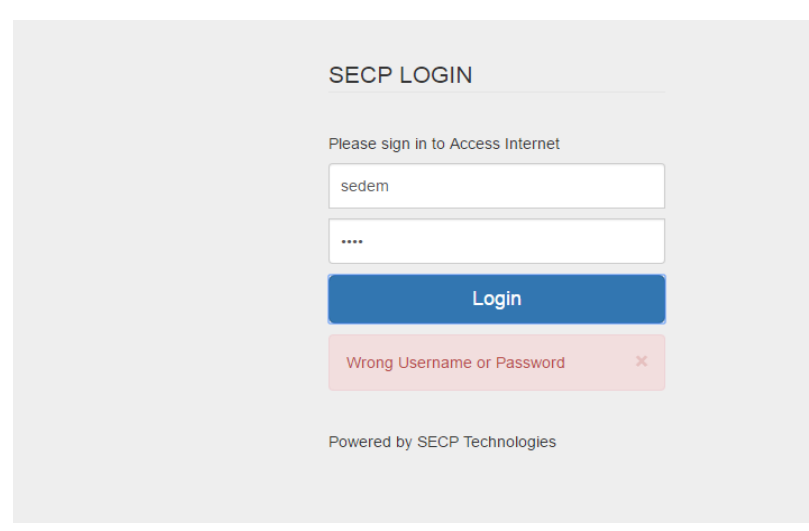

Figure 3: Screenshot of Error Login Page

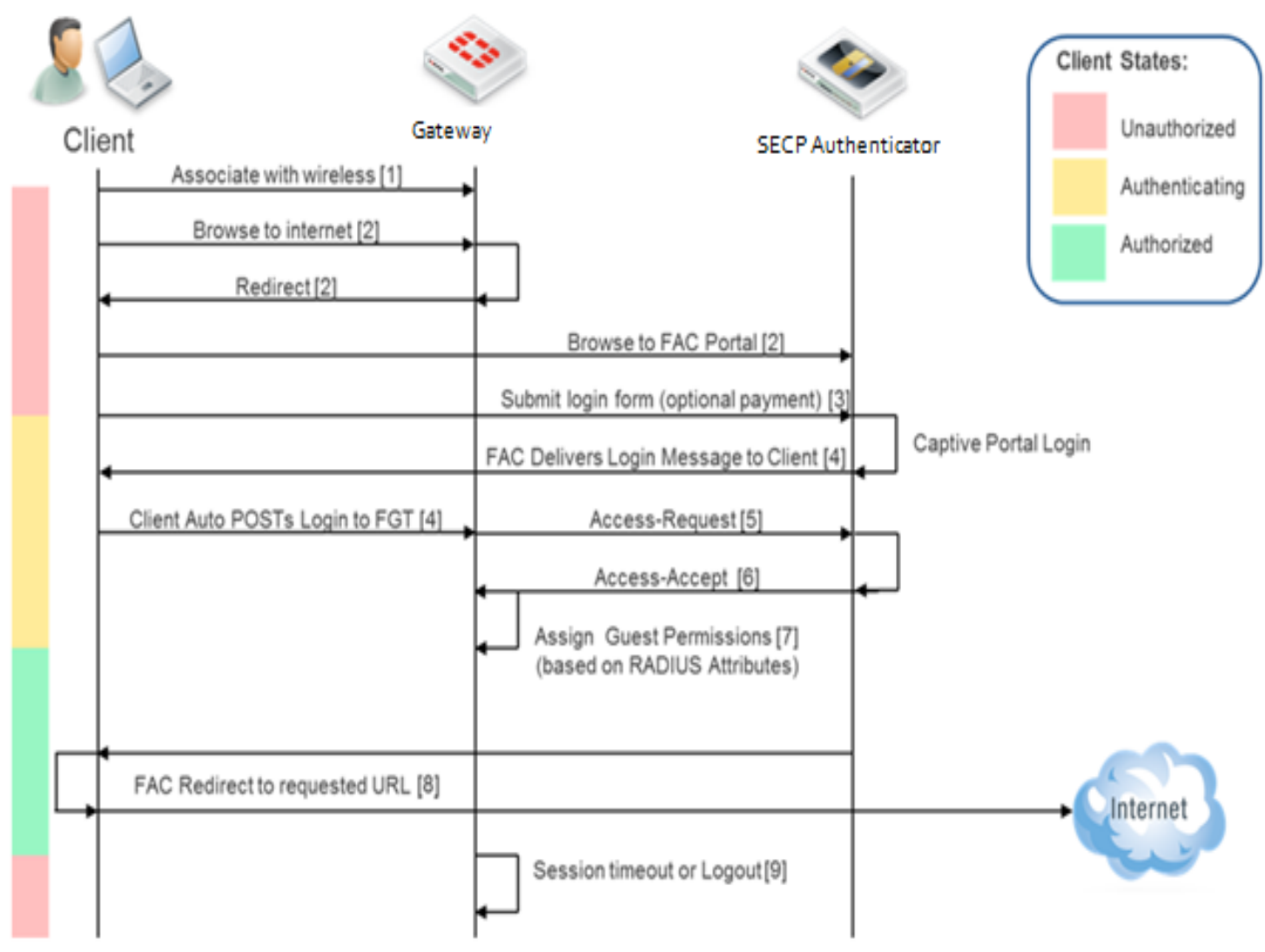

Figure 4: Sequential diagram for Proposed

\subsection{System Use Case Diagram}

A Use Case diagram is a graphic depiction of the interactions among the elements of a system. From the below Use Case Diagram of the proposed system, the rectangle is called System Boundary and the two diagrams outside the System Boundary are called Actors (Member/User and Administrator). System Boundary: involves everything or

\subsection{System Sequential Diagram}

The below sequential diagram for the proposed system shows the timeline and sequence, which is it shows the sequential timing of the system. From the diagram below, Client, Gateway and the System Authenticator are called objects. The vertical lines from the objects shows the timing sequentially.

Arrows: The arrows move from object to object (i.e. it moves sequentially from one object to the other). 


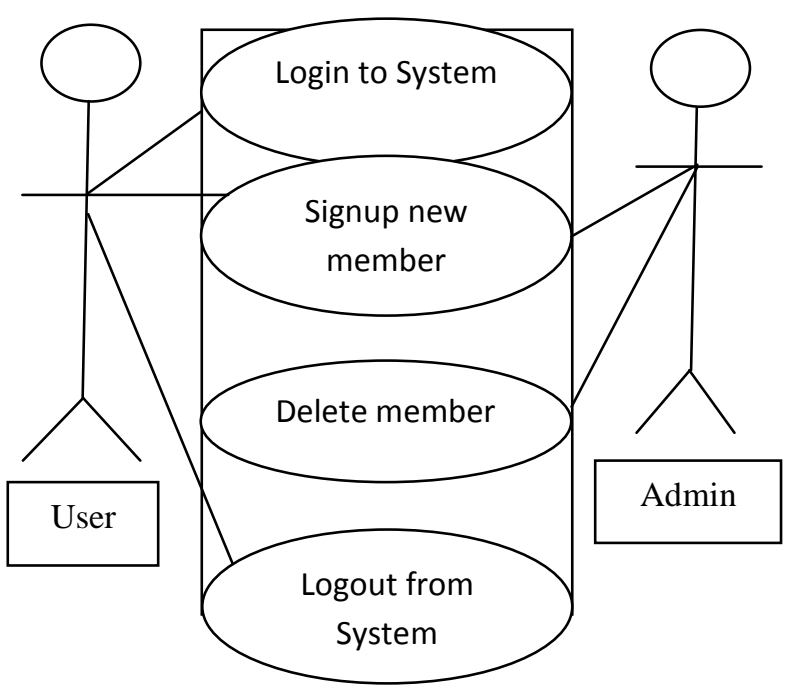

Figure 5: Use case diagram of the Proposed System

\subsection{Testing and Evaluation}

A unique website/web-based system contains two parts called authenticating users/Login page and Signup new members. Each part contains many forms. But out of them, the main forms or pages will be checked because of similarity and time constraint.

\subsubsection{Testing plan for Authenticating User (Login Page)}

The below table is used to test for errors and the login page of the system; it should show how the system detects login errors. The table consists of four columns. i.e. Number, Feature to be tested, Start Date and End Date. In the Feature to be tested column we have Login Page, Logout Page and Login Success Page. The Start date and End date are the start date for testing and end date for testing respectively.

Table 3: Testing plan for authenticating users

\begin{tabular}{|l|l|l|l|}
\hline No & Feature to be tested & Start date & End Date \\
\hline 1 & Login page & $13 / 05 / 2017$ & $13 / 05 / 2017$ \\
\hline 2. & Logout page & $14 / 05 / 2017$ & $14 / 05 / 2017$ \\
\hline 3 & Login success page & $15 / 05 / 2017$ & $15 / 05 / 2017$ \\
\hline
\end{tabular}

\subsubsection{Testing plan for Signup New Member}

The below table is used to test for errors and the signup page of the system; it should show how the system detects signup errors. The table consists of four columns. i.e. Number, Feature to be tested, Start Date and End Date. In the Feature to be tested column we have Signup Page, Signup Success Page and Username Exist Page. The Start date and End date are the start date for testing and end date for testing respectively. When a new member enters his/her credentials to sign up to the system, his or her credentials stores in the database and on the Signup Success Page short message is shown telling the new member that the signup is successful and an email will be sent to him shortly if verified.
Table 4: Testing plan for signup new members

\begin{tabular}{|l|l|l|l|}
\hline No & Feature to be tested & Start date & End Date \\
\hline 1 & Signup page & $13 / 05 / 2017$ & $13 / 05 / 2017$ \\
\hline 2. & Signup success page & $14 / 05 / 2017$ & $14 / 05 / 2017$ \\
\hline 3 & Username exist page & $15 / 05 / 2017$ & $15 / 05 / 2017$ \\
\hline
\end{tabular}

\subsection{Explanation of criteria used for testing}

Data: Information that needs to be entered at the form by the user

Procedure: Processing with the information as typed at the form

Expected Result: Describing or assuming what error will be displayed when the information is processed

Actual Result: Displaying the precise error message and suggesting for correction.

\subsubsection{Unit testing of the Proposed System}

The below table show what appears in the database when a new member is signup or registered weather verified or not verified. It shows the Username, Password, Email, Verified and Mod_timestamp. However, the password is hashed i.e. it is in a form of encrypted, the password of the user appears in a form of symbols, numbers and letters all together. The purpose of this unit testing is to test the member side of the signup new member form and also to test for dual username. Every username is unique and this system doesn't accept dual usernames.

The Expected Result of the unit testing of the system is "Username already exists" and Actual Result of the unit testing of the system is "Display error message to re-enter username".

\begin{tabular}{|c|c|}
\hline Purpose & $\begin{array}{l}\text { To test the member side of the signup } \\
\text { new members form }\end{array}$ \\
\hline Data & $\begin{array}{l}\text { Username : abdul } \\
\text { Password: } \\
\text { \$2y } \$ 10 \$ 0 \mathrm{cvHU} 5 \mathrm{GTLx} 7 \mathrm{cBVx62/qceeaH} \\
\text { UB1siLH58QL8dDkTUVz } \\
\text { Email: sakaradsalam@gmail.com } \\
\text { Verified: } 0 \\
\text { Mod_timestamp: 2017-06-13 03:56:54 }\end{array}$ \\
\hline Procedure & Type all data shown above \\
\hline $\begin{array}{l}\text { Expected } \\
\text { Result }\end{array}$ & Username already exists \\
\hline Actual Result & $\begin{array}{l}\text { Display error message to re-enter } \\
\text { username }\end{array}$ \\
\hline
\end{tabular}




\section{CONCLUSION}

This project considered a web-based captive portal system whose architecture consists of four main layers namely; Interactive layer, Logic layer, Access layer and Storage layer. The implementation was carried out using Adobe Dreamweaver and Xampp server. Following the completion of the review of the relevant literature and research conducted in Tamale Metropolis, it was realized that there is a need for the protection of Wireless Local Area Network in Tamale and Ghana as a whole. The core reasons for the establishment of the proposed system is to help reduce the security threats associated with Wireless Local Area Network, to allow/help wireless users protect their networks against hackers and to allow for Customizable Usage Plans. In conclusion, from proper analysis and assessment of the designed system it can be safely concluded that the system is an efficient, usable and reliable Captive Portal. The new system is expected to give benefits to the administrators (Network Administrators) in terms of efficiency in the system.

\section{REFERENCES}

[1] Kujala, M. (2003, May 28). WLAN standardsand Wireless networking security. Helsinki University of Technology Telecommunications Software and Multimedia Laboratory, 1-9.

[2] NetSol. (2007, June 16). netsolghana.com. Retrieved from NetSol Ghana Web site: hhtp://www.netsolghana.com

[3] Whitepapers. (2008, July 7). Wireless. Retrieved November 18, 2016, from support.usr.com: http://support.usr.com/download/whitepapers/wirelesswp.pdf
[4] Bradley, A. (2016, November 21). thoughtco. Retrieved June 15, 2017, from Why Use PHP?:http://php.about.com/od/phpbasics/p/php_reasons. htm.

[5] Sari, A., \& Karay, M. (2015). Impact of Determinants on Student Performance towards Information Communication Technology in Higher Education. International Journal of Learning and Development,, 2, 18-30.

[6] Select Business Solutions. (2014, April 11). selectbs.com/process-maturity/what-is-dsdm. Retrieved June 5, 2017, from selectbs.com http://www.selectbs.com/process-maturity/what-is-dsdm.

[7] Region, T. G. (2010, December 20). Wireless Networking Security. Retrieved February 24, 2017, from Analysis of "Hole 196" WPA2 Attack: https://airheads.arubanetworks.com/article/arubaanalysishole-196-wpa2-attack

[8] Karay, A. S. (2015, December). Comparative Analysis of Wireless Security Protocols: WEP vs WPA . Int. J. Communications, Network and System Sciences,, 8, 483491.

[9] Kumar, U. A. (2014). A Literature Review of Security Threats to Wireless Networks. International Journal of Future Generation Communication and Networking,, 7, 25-34.

[10] Chhillar, P. A. (2012, September-October). Review of WI-FI Security techniques . International Journal of Modern Engineering Research (IJMER), Vol. 2, Issue. 5,3479-3481. 